TPI-MINN-00/24 UMN-TH-1902-00 ITP-SB-00-20

\title{
Low-energy Limits on the Antisymmetric Tensor Field Background on the Brane and on the Non-commutative Scale
}

\author{
Irina Mocioiu ${ }^{1 *}$, Maxim Pospelov $^{2 \dagger}$ and Radu Roiban ${ }^{1 \ddagger}$ \\ ${ }^{1}$ C.N. Yang Institute for Theoretical Physics \\ State University of New York, Stony Brook, NY 11794-3840 \\ 2 Theoretical Physics Institute, School of Physics and Astronomy \\ University of Minnesota, 116 Church St., Minneapolis, MN 55455, USA
}

(November 14, 2018)

\begin{abstract}
A non-vanishing vacuum expectation value for an antisymmetric tensor field leads to the violation of Lorentz invariance on the brane. This violation is controlled by the $\theta_{\mu \nu}$ parameter, which has a dimension of inverse mass squared. We assume that the zeroth order term in $\theta$-expansion represents the Standard Model and study the effects induced by linear terms in $\theta_{\mu \nu}$. Low-energy precision experiments place the limit on the possible size of this background at the level of $1 / \sqrt{\theta} \gtrsim 5 \times 10^{14} \mathrm{GeV}$. This poses certain difficulty for the TeV-range string scale models, in which the antisymmetric field has to acquire a relatively large mass to ensure that $\theta_{\mu \nu}=0$ and avoid cosmological moduli problem.
\end{abstract}

\footnotetext{
*mocioiu@insti.physics.sunysb.edu

†pospelov@mnhepw.hep.umn.edu

${ }^{\ddagger}$ roiban@insti.physics.sunysb.edu
} 


\section{INTRODUCTION}

One of the initial motivations for field theories on non-commutative spaces was their intrinsically more convergent behavior in the ultraviolet regime than the one observed for ordinary field theories.

Field theories on noncommutative spaces (NCFT) can be defined as theories in their own right, independent of string theory. The coordinates in these spaces are represented by selfadjoint operators acting on some Hilbert space $\mathcal{H}$ and satisfying the following commutation relations:

$$
\left[\hat{x}^{\mu}, \hat{x}^{\nu}\right]=i \theta^{\mu \nu} \quad\left[\theta^{\mu \nu}, x^{\rho}\right]=0
$$

Consequently, fields on such a space are replaced by operators. To each such operator one can associate an ordinary field on a commutative space as follows:

$$
\phi(x)=\frac{1}{(2 \pi)^{d / 2}} \int d^{d} k \mathrm{e}^{i k_{\mu} x^{\mu}} \operatorname{Tr}\left[\hat{\phi}(\hat{x}) \mathrm{e}^{-i k_{\mu} \hat{x}^{\mu}}\right]
$$

where the trace is taken in the Hilbert space $\mathcal{H}$. By $\phi$ here we denote a generic field; we can associate to it some space-time indices as it will be the case for gauge fields or fermions.

An action defined on $\mathcal{H}$ must be naturally writable in terms of traces; furthermore, we want that in the limit $\theta^{\mu \nu} \rightarrow 0$ the expression reduces to an ordinary action on an ordinary, commutative space. Then, the generic form of the action is

$$
S=\operatorname{Tr}\left[\left(\theta_{\mu \nu}^{-1}\left[\hat{x}^{\nu}, \hat{\phi}(\hat{x})\right]\right)^{2}+P(\hat{\phi})\right]
$$

where $P$ is some polynomial in $\hat{\phi}(\hat{x})$. It is not difficult to see that, using (2), the commutation relations for $\hat{x}$ and the Baker-Campbell-Hausdorff formula, this action reduces to:

$$
S=\int d^{D} x\left(\partial_{\mu} \phi(x)\right)^{2}+P_{*}(\phi)
$$

where by $P_{*}(\phi)$ we mean that in $P(\hat{\phi})$ we replace $\hat{\phi}$ by $\phi$ and the product of fields is the Moyal product, given by:

$$
\phi_{1} * \phi_{2}(x)=\left.e^{i \frac{1}{2} \theta^{\mu \nu} \frac{\partial}{\partial \xi^{\mu}} \frac{\partial}{\partial \zeta^{\nu}}} \phi_{1}(x+\xi) \phi_{2}(x+\zeta)\right|_{\xi=\zeta=0}
$$

Noncommutative field theories became popular among string theorists with the work of Connes, Douglas and Schwarz [1] who argued that M-theory in constant 3-form background is equivalent to the supersymmetric Yang-Mills theory, defined on a non-commutative torus. A second wave of interest was generated by the paper of Seiberg and Witten [2] which summarized and extended earlier ideas about the appearance of noncommutative geometry in string theory with constant NS-NS $B$ field background.

NCFT are constructed starting from string theory in much the same way as the usual field theories. In particular, one computes the string theory S-matrix elements and writes a low energy effective action that reproduces them at the tree level. Furthermore, the only difference from the usual computation is that the world sheet propagator is modified by the presence of $B$. If the world sheet has no boundaries, then a constant $B$ field can 
be gauged away. Thus, a constant B flux manifests itself only in the presence of world sheet boundaries, i.e. in the presence of D-branes. Moreover, the same argument shows that only the components of $B$ parallel to the D-brane can be non-zero. The world sheet propagator restricted to the boundary is modified by the addition of a term $\frac{i}{2} \theta^{\mu \nu} \epsilon\left(\tau-\tau^{\prime}\right)$, where $\epsilon\left(\tau-\tau^{\prime}\right)$ is the step function and $\theta^{\mu \nu}=-\left(2 \pi \alpha^{\prime}\right)^{2}\left(\frac{1}{g+2 \pi \alpha^{\prime} B} B \frac{1}{g-2 \pi \alpha^{\prime} B}\right)^{\mu \nu}$. Since all vertex operators contain factors of the type $\exp (i k \cdot x)$, it is easy to see that all correlation functions will get the extra factor

$$
e^{i \theta^{\mu \nu}} \sum_{i<j=1}^{n} k_{\mu}^{i} k_{\nu}^{j}
$$

which is just the $*$-product defined in (5) written in momentum space. Thus, the effective action in the presence of B-field has the interpretation of a field theory on a noncommutative space with noncommutativity parameter given by $\theta$.

Transition from the open string theory to the non-commutative field theory becomes explicit in a zero slope limit, $\alpha^{\prime} \sim \epsilon^{1 / 2} \rightarrow 0, g_{\mu \nu} \sim \epsilon \rightarrow 0$. For example, in this limit, the dynamics of Yang-Mills fields living on the brane is governed by the following action:

$$
S=\frac{1}{2 g_{Y M}^{2}} \int \operatorname{Tr}_{U(N)} F_{\mu \nu}^{n c} * F^{n c, \mu \nu}
$$

where we have taken the open string metric to be $\eta_{\mu \nu}$ and $F_{\mu \nu}^{n c}$ is given by:

$$
F_{\mu \nu}^{n c}=\partial_{\mu} A_{\nu}^{n c}-\partial_{\nu} A_{\mu}^{n c}+i A_{\mu}^{n c} * A_{\nu}^{n c}-i A_{\nu}^{n c} * A_{\mu}^{n c} .
$$

Comparing this with equation (国) we see that this can be interpreted as the Yang-Mills action on a noncommutative space with noncommutativity parameter $\theta$. Up to now the structure of $B_{\mu \nu}$ in the directions parallel to the brane was not really important. It should be noted, however, that large electric-like background $B_{0 i}$ creates various problems in the zero slope limit [3], 国. A solution to these problems was proposed in [4] and it leads to (7).

It became a colloquial wisdom that the parameter $\theta_{\mu \nu}$ does not necessarily have to be of the order of the inverse Plank scale squared [2,5]. For example, $\theta_{\mu \nu}$ could be significantly larger in the "brane-world" proposal [6], in which the string scale $M_{s}$ is much smaller than four-dimensional Plank scale due to the large volume of extra dimensions, $M_{\mathrm{Pl}}^{2}=M_{s}^{n+2} V_{n}$. In this case a "natural" scale for $\theta_{\mu \nu}$ could be $M_{s}^{-2}$. Another example is the open string realization of the non-commutative field theories on the brane in the zero slope limit described above, which presumes the non-commutative scale to be fixed, while gravity is decoupled (i.e. $\left.M_{\mathrm{Pl}} \rightarrow \infty\right)$. If instead we choose to fix the gravitational scale, zero slope limit would correspond to a large non-commutative parameter in units of $M_{\mathrm{Pl}}^{-2}$.

All these cases pose one interesting phenomenological question as to how large $\theta_{\mu \nu}$ could be without contradicting existing experimental data. To answer this question we have to adopt certain calculational framework, incorporating together Standard Model fields and $\theta_{\mu \nu}$ background. The simplest way of doing this is to assume that SM is realized on the brane and the external background of $B_{\mu \nu}\left(\right.$ or $\left.\theta_{\mu \nu}\right)$ field is included via Moyal product. In this paper we take space and time independent background which could be a bad approximation at large distances. Indeed, due to the interaction on the brane, $B_{\mu \nu}(x)$ could become a massive field so that the minimum of energy correspond to the vanishing vev. In this case 
the constraints on $\theta_{\mu \nu}$ that we are aiming to produce will be trivially satisfied since there is no $\theta$ to begin with and the theory is the usual one. Despite this possibility, we believe that the question of experimental constraints on $B_{\mu \nu}$ background/noncommutativity parameter $\theta_{\mu \nu}$ deserves special investigation.

Our strategy for the rest of this paper is quite straightforward. We take the $\left(^{*}\right)$-modified Standard Model and expand it once in the external $\theta$ parameter,

$$
f * g=f g+\frac{i}{2} \theta_{\mu \nu} \partial_{\mu} f \partial_{\nu} g
$$

so that SM is extended by the series of dimension 6 operators, composed from three or more fields:

$$
S M(*)=S M+\sum_{i} \theta_{\mu \nu} O_{\mu \nu}^{(i)}
$$

Here the summation is performed over different types of operators, full list of which is outside the scope of the present paper. First order in $\theta$ is sufficient for our purposes. Such a procedure of constructing an effective action does not depend on taking the zero slope limit and holds for a generic situation [2].

It is also to our advantage that in the effective Lagrangian approach we do not have to worry about the renormalizability of this theory. Although the ultimate resolution to the question of renormalizability of non-commutative field theories is very interesting and very important [7], here we can simply assume that all loop divergences are regularized at momenta comparable to $M_{s}$. Moreover, since the natural scale for $\theta$ is $M_{s}^{-2}$, the iteration of $\theta_{\mu \nu} O_{\mu \nu}$-interactions does not create any problems as long as external momenta are much smaller than $\sqrt{1 / \theta}$.

\section{EXPERIMENTAL LIMITS ON $\theta_{\mu \nu}$}

\section{High-energy limits}

At first glance, it is advantageous to use high-energy processes to obtain the most stringent limits on $\theta$. Indeed, the higher the energy/momentum transfer is, the larger the effect of $O_{\mu \nu}$ will be. Let us consider such a well-studied process as the Z-boson decay. We shall profit from the fact that $O_{\mu \nu}$ violate Lorentz invariance and use a decay channel which is strictly forbidden at the SM level and allowed when $\theta_{\mu \nu} \neq 0$. A good candidate for this channel will be the decay of $\mathrm{Z}$ into a pair of photons, forbidden by Lorentz invariance and Bose statistics in SM. The experimental limit on the branching ratio for this decay is also very good, $\operatorname{Br}(Z \rightarrow \gamma \gamma) \leq 5 \cdot 10^{-5}[8]$.

To calculate this decay width we need to evaluate the $\theta_{\mu \nu} O_{\mu \nu}$-expansion of the $U(1)$ gauge sector, which in the presence of external $\theta_{\mu \nu}$-background could be taken in the following form:

$$
S_{n c}=-\frac{1}{4} \int d^{4} x F_{\mu \nu}^{n c} * F^{n c, \mu \nu}
$$

Here $F_{n c}$ denotes "non-commutative" field strength given by

$$
F_{\mu \nu}^{n c}=\partial_{\mu} B_{\nu}-\partial_{\nu} B_{\mu}-g^{\prime} \theta^{\alpha \beta} \partial_{\alpha} B_{\mu} \partial_{\beta} B_{\nu}
$$


Expanding Eq. (11) to first order in $\theta_{\mu \nu}$ we get

$$
S_{n c}=S+\frac{1}{2} g^{\prime} \int d^{4} x \theta^{\alpha \beta}\left(\partial_{\mu} B_{\nu}-\partial_{\nu} B_{\mu}\right) \partial_{\alpha} B^{\mu} \partial_{\beta} B^{\nu}
$$

Going to the physical basis, we obtain the $Z \gamma \gamma$ interaction term:

$$
S_{\text {int }}=\frac{1}{2} g^{\prime} \sin \theta_{W} \cos ^{2} \theta_{W} \int d^{4} x \theta_{\rho \sigma}\left[\partial_{\mu} Z_{\nu} \partial_{\rho} A_{\mu} \partial_{\sigma} A_{\nu}+\left(\partial_{\mu} A_{\nu}-\partial_{\nu} A_{\mu}\right) \partial_{\rho} Z_{\mu} \partial_{\sigma} A_{\nu}\right] .
$$

It should be noted here that the expansion of the $S U(2)$ sector has quadratic terms in $\theta_{\mu \nu}$, but not linear ones and thus does not contribute into (14).

Finally, we arrive at the following answer for the two-photon decay width of the Z-boson, induced by the $\theta_{\mu \nu}$-background:

$$
\Gamma_{Z \rightarrow \gamma \gamma}=\frac{\alpha}{144} \cos ^{4} \theta_{W} M_{Z}^{5} \sum_{i} \theta_{0 i}^{2} .
$$

The decay width is evaluated in the Lorentz system in which $\mathrm{Z}$ is produced at rest.

Comparing this result with the experimental limits on the branching ratio, we conclude that the sensitivity to $1 / \sqrt{\theta}$ is not better than $250 \mathrm{GeV}$. This is a very modest limit, which could perhaps be improved had we considered $\theta$-induced corrections to other high-energy processes such as $e^{+} e^{-}$cross sections, forward-backward asymmetry and so on. At any rate, a significant improvement beyond the level of $250 \mathrm{GeV}$ is not possible. Thus, we conclude that the high-energy processes cannot produce sufficiently strong bounds on $\theta_{\mu \nu}$, and turn to the low-energy limits on this parameter.

\section{Low-energy limits}

The most notable feature of the effective Lagrangian (10) is the explicit violation of Lorentz invariance [9]. This violation is, of course, controlled by the size of $\theta_{\mu \nu}$ and could be made arbitrarily small "by hand".

The extension of Standard Model by Lorentz-noninvariant operators of dimension $\leq 4$, has been actively studied in the past [10]. Some of the limits obtained in 10] are extremely strong. A priori, there are several problems with such a generic description. If we believe that the Lorentz non-invariant terms originate from short distances, it is not clear why dimension 3 and 4 operators should be suppressed. Another problem is of rather technical nature, as the number of free parameters in such extensions of SM is generally very large. These problems do not exist in our approach. The breaking of Lorentz invariance is given only by $\theta_{\mu \nu}$, and its effect first shows up in dimension 6 operators.

The qualitative understanding of the role of $\theta_{\mu \nu}$ for low-energy physics comes from the considerations of the non-relativistic limit for the $\int d^{4} x e \bar{\psi} * A_{\mu} \gamma^{\mu} * \psi$ interaction term taken in the external Coulomb field:

$$
V=-\frac{Z \alpha}{r}-\frac{Z \alpha}{2 r^{3}}\left(\theta_{B} \cdot \mathbf{L}\right)-\frac{m_{e} Z \alpha}{r^{3}}\left(\theta_{E} \cdot \mathbf{r}\right)
$$

Here we split $\theta_{\mu \nu}$ into two three-vectors in analogy with the electromagnetic field, $\epsilon_{i j k}\left(\theta_{B}\right)_{k} \equiv$ $\theta_{i j},\left(\theta_{E}\right)_{i} \equiv \theta_{0 i}$. The diagonal matrix element of the last operator in Eq. (16), taken over a wave function of the discrete spectrum, is equal to zero, unless $\mathrm{CP}$ is broken. The second 
term, however, may produce interesting phenomena, as it gives an effective coupling of the angular momentum with an external vector $\theta_{B}$,

$$
V=\kappa\left(\theta_{B} \cdot \mathbf{J}\right)
$$

The effective coupling constant $\kappa$ has obvious $L, J$ dependence and, more importantly, is determined by the third power of the characteristic atomic momentum. For an outer atomic electron this coupling is of the order of $Z^{2} \alpha\left(m_{e} \alpha\right)^{3}$. The coupling to $\theta_{B}$ creates a Zeemanlike splitting of atomic orbitals with respect to an external vector and thus can be tested in high-precision atomic experiments.

At this point it becomes clear that similar effects $\left(\theta_{B} \cdot\right.$ angular momentum) will appear in the hadronic physics, when we consider the $(*)$-extended interaction of quarks and gluons. This interaction will lead to the effective coupling of the nucleon spin with $\theta$,

$$
\left\langle N\left|\mathcal{L}_{Q C D}(*)\right| N\right\rangle=\theta \text {-independent terms }+\frac{d_{\theta}}{2} \theta_{\mu \nu} \bar{N} \sigma_{\mu \nu} N
$$

which in non-relativistic limit simply becomes $d_{\theta}\left(\theta_{B} \cdot \frac{\mathbf{S}}{S}\right)$. The size of $d_{\theta}$ is given by a cube of a characteristic hadronic scale.

To estimate $d_{\theta}$ and to convince ourselves that such an effect exists, we perform the following exercise, a simplified version of the nucleon three-point function QCD sum rules [11]. We calculate the operator product expansion (OPE) of two nucleon currents in the presence of the external $\theta_{\mu \nu}$-background and observe the non-zero result. Then we take the "phenomenological" part of the QCD sum rule and saturate it with the nucleon double-pole contribution. Matching the two sides at $1 \mathrm{GeV}$, we obtain an estimate of $d_{\theta}$ for nucleons.

On the OPE side we can use an asymptotically free description, and thus include the $\theta_{\mu \nu}$-piece as the correction to a free massless quark propagator:

$$
S(x, 0)=\frac{i x_{\mu} \gamma_{\mu}}{2 \pi^{2} x^{4}}-\frac{i x_{\mu} \gamma_{\beta}}{2 \pi^{2} x^{4}} t^{a} G_{\nu \beta}^{a} \theta_{\mu \nu}
$$

This correction originates from the $\left(^{*}\right)$-extended quark-gluon interaction, whereas pure gluonic sector does not contain terms linear in theta. Using this propagator it is straightforward to calculate the OPE of two nucleon currents, expressing the result as the combination of a pure perturbative piece and non-perturbative condensates such as $\left\langle\bar{q} \sigma_{\mu \nu} q\right\rangle_{\theta_{\mu \nu}},\left\langle\bar{q} \sigma_{\mu \nu} t^{a} G_{\mu \nu}^{a} q\right\rangle$, and so on. Deferring further details for more extended publication, we present here the final estimate for $d_{\theta}$ :

$$
d_{\theta} \sim 0.1 \mathrm{GeV}^{3}
$$

Further progress in refining this estimate can be achieved by including the anomalous dimensions, and fixing the size of "susceptibility" condensate $\left\langle\bar{q} \sigma_{\mu \nu} q\right\rangle_{\theta_{\mu \nu}}$ by comparing the stability of different sum rule channels.

As we remarked earlier, the coupling of the nucleon spin to an external vector will lead to the Zeeman-like splitting of the hyperfine structure in atoms. Since the precision in measuring hyperfine splittings is at the level of $\mathrm{mHz}$, this should lead to a strong bound on $\theta_{B}$. One of the most sensitive systems in this respect is the hydrogen maser, where the effects of Lorentz violating terms have been searched for in a recent experiment [12]. In this 
double-resonance experiment the absence of sidereal variations of the maser frequency leads to $1 \mathrm{mHz}$ limit on the product of $d_{\theta}$ and $\theta_{B \perp}$, component of $\theta_{B}$ perpendicular to earth's axis. This puts the limit on the magnetic component of $\theta_{\mu \nu}$ at the level $1 / \sqrt{\theta}>5 \cdot 10^{12} \mathrm{GeV}$.

Another even more stringent limit could be extracted from the experiment which compares magnetic field measured by $\mathrm{Cs}$ and $\mathrm{Hg}$ atoms [13]. The non-vanishing $\theta_{\mu \nu}$-background affects primarily nuclear spin. Thus the magnetic field, measured by mercury atom, will be corrected due to the interaction of the nuclear spin with $\theta_{B}$ to a larger extent than the magnetic field measured by Cs. The absence of sidereal variations in the difference of magnetic field measured by $\mathrm{Cs}$ and $\mathrm{Hg}$ is verified at $100 \mathrm{nHz}$ level. This translates to the following, extremely tight bound on $\theta$ :

$$
\frac{1}{\sqrt{\theta}}>5 \cdot 10^{14} \mathrm{GeV}
$$

This is the main result of the present paper.

\section{DISCUSSION}

The limit on $\theta_{\mu \nu}$ obtained in this paper is quite strong, signaling the sensitivity of lowenergy experiments to the scales comparable to standard GUT/string scales. Of course, this sensitivity is the consequence of the assumption about non-vanishing $\theta_{\mu \nu}$-dependent background, which breaks explicitly Lorentz invariance.

Limit (21) is derived from the atomic experiments, performed in a laboratory. It would be interesting to explore whether similar or stronger bounds could be obtained from nonobservation of the anisotropy of the Universe at large scales. Indeed, the coupling of baryon spins to $\theta_{\mu \nu}$ at certain level should lead to the anisotropy of matter distribution and polarization of interstellar medium which could, in principle, result in strong bounds on $\theta_{\mu \nu}$ [14.

Returning to the brane-world scenario it is fair to question a "natural" value for $\theta_{\mu \nu}$ on the brane. For the energies much higher than the inverse radius of extra dimensions, a natural "string" value of $\theta_{\mu \nu}$ is given by the square of the inverse string scale. 円 For $M_{s} \sim$ $1 \mathrm{TeV}$ this is in apparent contradiction with the limit (21).

Does our result (21) pose another "naturalness" problem for the low-energy string scale models? The answer to this question depends on the dynamical properties of $\theta_{\mu \nu}(x, y)$, where $y$ indicates the dependence on extra space-like coordinates. If the lowest mode of the Kaluza-Klein expansion of $\theta_{\mu \nu}(x, y)$ remains essentially massless, as it would happen in theories with extra dimensions of infinite volume [15], then the vev of $\theta_{\mu \nu}(x, y)$ may freeze at a large value, which would be in contradiction to the observational evidence for absence of $\theta_{\mu \nu}$ at the level (21). When the volume of extra dimensions is finite, it may turn out that the ultimate value of $\theta_{\mu \nu}(x)$ is zero. Indeed, every Kaluza-Klein copy of this tensor field may receive a mass term due to the interaction with brane fields and/or details of mechanisms

1 Throughout our discussion we do not assume zero slope limit, so that $M_{s}$ is the only natural dimensional high-energy parameter. 
responsible for compactification. The mass term induced by the interaction $\theta_{\mu \nu} O_{\mu \nu}$ on the brane could be estimated when the volume of extra dimensions is large. If we assume that the correlator of two $O_{\mu \nu}$ currents is saturated at the string scale $M_{s}$,

$$
\int\langle O(x), O(0)\rangle d^{4} x \sim(\text { two loop factor }) \times M_{s}^{4},
$$

and the two-loop phase space factor originates from the fact that by construction every $O_{\mu \nu}$ has at least three fields. The kinetic term for every Kaluza-Klein mode of $\theta_{\mu \nu}(x, y)$ receives a volume enhancement factor, so that the effective mass for the lowest mode, canonically normalized in four dimensions is

$$
m_{\text {eff }}^{2} \sim(\text { two loop factor }) \times \frac{M_{s}^{2}}{M_{s}^{n} V_{n}} \sim 10^{-4} \times \frac{M_{s}^{4}}{M_{\mathrm{Pl}}^{2}} \sim\left(10^{-5} \mathrm{eV}\right)^{2} \frac{M_{s}^{2}}{1 \mathrm{TeV}^{2}}
$$

It is clear that for a $\mathrm{TeV}$ range $M_{s}^{2}, m_{e f f}$ is very low, which could create cosmological problems, similar to those arising in the standard axion relaxation mechanism when the axion mass is small. In this case the " $\theta_{\mu \nu}$-problem" is replaced by a new moduli problem which indicates that the choice $M_{s} \sim 1 \mathrm{TeV}$ is unnatural. However, the ultimate answer to the question about the mass of $\theta_{\mu \nu}(x)$ and its vev, surviving until present cosmological times, needs other physical inputs such as information about the compactification mechanisms and supersymmetry breaking.

Acknowledgments M.P. thanks A. Lossev for very helpful discussions and interest taken in this work. I.M. and R.R. thank I. Chepelev for discussions. This work was supported in part by the Department of Energy under Grant No. DE-FG02-94ER40823 and NSF grant PHY-9722101. 


\section{REFERENCES}

[1] A.Connes, M.R. Douglas, and A. Schwarz, JHEP 9802 (1998) 003

[2] N. Seiberg and E. Witten, JHEP 9909 (1999) 032

[3] N. Seiberg, L. Susskind, N. Toumbas, hep-th/0005040; R. Gopakumar, J. Maldacena, S. Minwalla, and A. Strominger, hep-th/0005048.

[4] J.L.F. Barbon, E. Rabinovici, hep-th/0005073.

[5] J. Madore, gr-qc/9906059.

[6] N. Arkani-Hamed, S. Dimopoulos, and G. Dvali, Phys. Lett. B429 (1998) 263; I. Antoniadis, N. Arkani-Hamed, S. Dimopoulos, and G. Dvali, Phys. Lett. B436 (1998) 257; N. Arkani-Hamed, S. Dimopoulos, and G. Dvali, Phys. Rev. D59 (1999) 086004.

[7] C.P. Martin and D. Sanchez-Ruiz, Phys.Rev.Lett. 83 (1999) 476, hep-th/9903077; M.M. Sheikh-Jabbari, JHEP 9906 (1999) 015, hep-th/9903107; T. Krajewski and R. Wulkenhaar, hep-th/9903187; I. Chepelev, R. Roiban, hep-th/9911098; S. Minwalla, M. Van Raamsdonk, N. Seiberg, hep-th/9912072; I. Ya. Aref'eva, D. M. Belov, A. S. Koshelev, hep-th/0001215; H. Grosse, T. Krajewski, R. Wulkenhaar, hepth/0001182; J. Ambjorn, Y.M. Makeenko, J. Nishimura, R.J. Szabo, hep-th/0004147.

[8] C. Caso et al, The Eur. Phys. J. C3 (1998) 1.

[9] For other realization of the violation of Lorentz invariance on the brane see G. Dvali and M. Shifman, Phys. Rept. 320 (1999) 107.

[10] D. Colladay, V.A. Kostelecky, Phys. Rev. D58 (1998) 116002; S. Coleman and S.L. Glashow, Phys. Rev. D59 (1999) 116008; V.A. Kostelecky and C.D. Lane, Phys. Rev. D60 (1999) 116010; R. Bluhm, V.A. Kostelecky, and N. Russell, hep-ph/0003223.

[11] For similar calcuations of the magnetic and electric dipole moments of nucleons see, for example, B.L. Ioffe and A.V. Smilga, Nucl. Phys. B232 (1984) 109; I.I. Balitsky and A.V. Yung, Phys. Lett. B129 (1983) 328; M. Pospelov and A. Ritz, Phys. Rev. Lett. 83 (1999) 2526; M. Pospelov and A. Ritz, Nucl. Phys. B573 (2000) 177.

[12] R.L. Walsworth and D.F. Phillips, to appear in the proceedings of International Conference on Orbis Scientiae 1999: Quantum Gravity, Generalized Theory of Gravitation and Superstring Theory Based Unification (28th Conference on High Energy Physics and Cosmology Since 1964), Fort Launderdale, Florida, 16-19 Dec 1999.

[13] C.J. Berglund et al, Phys. Rev. Lett. 75 (1995) 1879.

[14] S.M. Carroll, G.B. Field, and R. Jackiw, Phys. Rev. D41 (1990) 1231.

[15] R. Gregory, V.A. Rubakov, and S.M. Sibiryakov, hep-th/0002072; G. Dvali, G. Gabadadze, and M. Porrati, hep-th/0005016. 